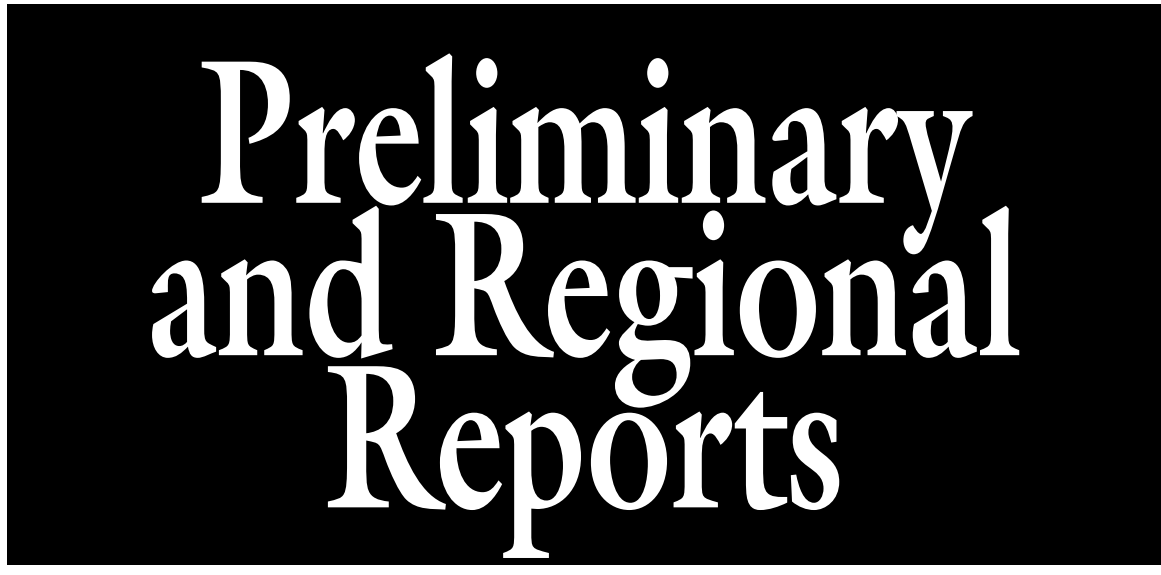

\section{Effect of Soil Type and Nitrogen Rate on Growth of Annual and Perennial Landscape Plants in Florida}

\author{
Kimberly A. Moore ${ }^{1,5,8}$, Amy L. Shober ${ }^{2,6}$, Gitta Hasing ${ }^{3,7}$, \\ Christine Wiese $^{4,7}$, and Nancy G. West ${ }^{3,7}$
}

ADDITIONAL INDEX WORDs. soil properties, ornamental plants

SuMmary. Previous research indicated that acceptable quality annual and perennial plant species can be grown in the landscape with low nitrogen $(\mathrm{N})$ inputs. However, information on the impact of soil conditions and $\mathrm{N}$ use by ornamental plants grown in central Florida is lacking in the literature. Our objective was to evaluate plant growth and quality response of eight warm-season annuals, seven cool-season annuals, and four herbaceous perennial species to a range of $\mathbf{N}$ fertilizer rates when plants were grown in landscape beds containing native field soil or subsoil fill. A slow-release $\mathrm{N}$ source $(42 \mathrm{~N}-$ $0 \mathrm{P}-0 \mathrm{~K}$ ) was applied every 12 weeks at annual $\mathrm{N}$ rates of 3,5 , or $7 \mathrm{lb} / 1000 \mathrm{ft}^{2}$ for a period of 18 weeks (annual species) or 1,3 , or $5 \mathrm{lb} / 1000 \mathrm{ft}^{2}$ for a period of 54 weeks (perennial species). Plants were evaluated for aesthetic quality every 6 weeks and shoot dry weight was measured at completion of the experiment. Dry weight production and aesthetic quality of most species evaluated was unaffected by $\mathrm{N}$ rate. For several species, shoot dry weight was higher when planted in the field plots containing native soil [alyssum (Lobularia maritima) 'Bada Bing White' wax begonia (Begonia $\times$ semperflorens-cultorum), dahlberg daisy (Thymophylla tenuiloba), 'Survivor Hot Pink' geranium (Pelargonium $\times$ hortorum), gomphrena (Gomphrena globosa), 'Blue Puffs Improved' ('Blue Danube') ageratum (Ageratum houstonianum), blanket flower (Gaillardia pulchella), goldenrod (Solidago chapmanii), 'Mystic Spires' salvia (Salvia longispicata $\times$ farinacea)]. Quality response to soil condition was mixed over the course of the study. Several species performed as well (or better) in the field as when planted in the subsoil fill soils. These results illustrate that some landscape plant species are able to survive and thrive under various soil and fertility conditions. These "tougher" species may be good choices for installation in landscapes with marginal native soils or disturbed urban landscape soils. leach below the root zone during high intensity irrigation and precipitation events and contaminate groundwater systems. Florida's soils tend to be coarse-textured with low fertility and low water-holding capacity (Broschat et al., 2008). As a result, supplemental nutrients are easily leached downward through the soil profile. To insure that excess fertilizer is not applied to landscapes, accurate and specific fertilizer recommendations for ornamental plants are needed.

Previous research has suggested that the range of appropriate annual $\mathrm{N}$ fertilizer rates for producing acceptable growth and quality of landscapegrown plants was 4 to $6 \mathrm{lb} / 1000 \mathrm{ft}^{2} \mathrm{~N}$ for warm- and cool-season annuals (Shurberg et al., 2012b), and 2 to 4 $\mathrm{lb} / 1000 \mathrm{ft}^{2} \mathrm{~N}$ for herbaceous perennials (Shurberg et al., 2012a), shrubs (Shober et al., 2013), and vines and groundcovers (Shober et al., 2014). However, these $\mathrm{N}$ fertilizer ranges were determined under low fertility conditions (planted in sandy subsoil fill material). Therefore, it is likely that specific $\mathrm{N}$ fertilizer needs of annual and perennial landscape species will change when soil conditions, such as texture and fertility, vary. Our objective was to evaluate plant growth and quality response of warm- and coolseason annuals and herbaceous perennials to a range of $\mathrm{N}$ fertilizer rates when plants were grown in landscape beds containing native field soil or sandy subsoil fill.

\section{Materials and methods}

Plant material. Seven coolseason annuals, eight warm-season annuals, and four herbaceous perennial landscape plants were selected for evaluation across $\mathrm{N}$ rates and soil conditions.Cool-season annuals included alyssum, 'Bada Bing White' wax
$\mathrm{M}$ any homeowners that strive to attain aesthetically pleasing landscapes will err on the side of over application of fertilizer rather than insufficient application (Israel and Knox, 2001). However, there are potential environmental consequences of $\mathrm{N}$ fertilizer applications. Improperly applied fertilizer can be carried by water directly to surface water bodies via storm drains (Line et al., 2002). Excess nutrients may also

\begin{tabular}{llll}
\hline $\begin{array}{l}\text { Units } \\
\text { To convert U.S. to SI, } \\
\text { multiply by }\end{array}$ & U.S. unit & SI unit & $\begin{array}{l}\text { To convert SI to U.S., } \\
\text { multiply by }\end{array}$ \\
\hline 10 & $\%$ & $\mathrm{~g} \cdot \mathrm{kg}^{-1}$ & 0.1 \\
0.3048 & $\mathrm{ft}$ & $\mathrm{m}$ & 3.2808 \\
3.7854 & $\mathrm{gal}$ & $\mathrm{L}$ & 0.2642 \\
2.54 & inch $(\mathrm{es})$ & $\mathrm{cm}$ & 0.3937 \\
25.4 & inch $(\mathrm{es})$ & $\mathrm{mm}$ & 0.0394 \\
48.8243 & $\mathrm{lb} / 1000 \mathrm{ft}^{2}$ & $\mathrm{~kg} \cdot \mathrm{h}^{-1}$ & 0.0205 \\
1 & $\mu \mathrm{mho} / \mathrm{cm}$ & $\mu \mathrm{S} \cdot \mathrm{cm}^{-1}$ & 1 \\
28.3495 & $\mathrm{oz}$ & $\mathrm{g}$ & 0.0353 \\
1 & $\mathrm{ppm}$ & $\mathrm{mg} \cdot \mathrm{kg}^{-1}$ & 1 \\
1 & $\mathrm{ppm}$ & $\mathrm{mg} \cdot \mathrm{L}^{-1}$ & 1 \\
$\left({ }^{\circ} \mathrm{F}-32\right) \div 1.8$ & ${ }^{\circ} \mathrm{F}$ & ${ }^{\circ} \mathrm{C}$ & $\left({ }^{\circ} \mathrm{C} \times 1.8\right)+32$
\end{tabular}

Hortlechnology · December 2014 24(6) 
begonia, dahlberg daisy, 'Survivor Hot Pink' geranium, gomphrena, 'Super Elfin Bright Orange' impatiens (Impatiens walleriana), and 'Montego Yellow' and 'Montego Purple' snapdragon (Antirrbimum majus). Warm-season annuals species included 'Blue Puffs Improved' ('Blue Danube') ageratum, 'Cosmic Mix' and 'Cosmic Orange' cosmos (Cosmos sulphureus), 'Silverdust' dusty miller (Senecio cineraria), 'Bonanza Orange' marigold (Tagetes patula), 'Sundial Yellow' moss rose (Portulaca grandiflora), 'Saratoga Red' nicotiana (Nicotiana alata), 'Vista Red' salvia (Salvia splendens), and 'Profusion Cherry' zinnia (Zinnia elegans $\times$ angustifolia). Herbaceous perennial plant species included blanket flower, goldenrod, mondo grass (Ophiopogon japonicus), and 'Mystic Spires' salvia.

Annual species were received as plugs from Knox Nursery (Winter Garden, FL), with the exception of impatiens, wax begonia, and geranium, which were received in 4 -inch containers from American Farms Nursery (Naples, FL). Cool- and warm-season annuals received as plugs were grown in 4-inch azalea pots (Reb Plastic, Orlando, FL) for 6 weeks before transplanting into the garden. During the growing phase, annual species were fertilized with a liquid $21 \mathrm{~N}-3.2 \mathrm{P}-$ $5.8 \mathrm{~K}$ fertilizer (Peter's Professional; Everris NA, Dublin, OH). Cool-season annuals were fertilized at a rate of 100 ppm N twice per week for 1 week, then at a rate of $200 \mathrm{ppm} \mathrm{N}$ until transplanting. Warm-season annual species were fertilized during the growing phase at a rate of $250 \mathrm{ppm} \mathrm{N}$ twice per week until transplanting. Perennial species were received as 1 -gal containers from Sweetbay Nursey (Parrish, FL) or Landscape Flower Growers

\footnotetext{
${ }^{1}$ Environmental Horticulture Department, University of Florida, Institute of Food and Agricultural Science, Fort Lauderdale Research and Education Center, 3205 College Avenue, Davie, FL 33314

${ }^{2}$ Department of Plant and Soil Sciences, University of Delaware, 531 South College Avenue, Newark, DE 19716

${ }^{3}$ University of Florida, Institute of Food and Agricultural Science, Gulf Coast Research and Education Center, 14625 CR 672, Wimauma, FL 33598

${ }^{4}$ University of Florida, Institute of Food and Agricultural Science, P.O. Box 110675, Gainesville, FL 32611

${ }^{5}$ Professor

${ }^{6}$ Associate Professor

${ }^{7}$ Biologist

${ }^{8}$ Corresponding author. E-mail: klock@ufl.edu.
}

(Riverview, FL) and were transplanted without any further growing phase.

EXPERIMENTAL DESIGN. Eighteen raised-bed plots containing subsoil fill material (St. John's fine sand; Sandy, siliceous, hyperthermic Typic Alaquods) and 18 field plots containing native field soil [a mixture of Zolfo fine sand (Sandy, siliceous, hyperthermic Oxyaquic Alorthods) and Seffner fine sand (sandy, siliceous, hyperthermic Aquic Humic Dystrudepts)] [U.S. Department of Agriculture (USDA), 2004] were established at the University of Florida, Institute of Food and Agricultural Science Gulf Coast Research and Education Center in Wimauma, FL (USDA hardiness zone 9a).

Sixteen plants of each annual species were transplanted into clusters in each raised bed and field plot. Four clusters of four plants (planted in a square configuration with plants spaced $1 \mathrm{ft}$ apart) of each species were placed into nine raised beds $(10 \times 40 \times$ $0.5 \mathrm{ft})$ and nine field plots $(10 \times 31 \mathrm{ft})$ on 29 Oct. 2010 (cool season) and 25 May 2011 (warm season). A slowrelease $\mathrm{N}$ source $[42 \mathrm{~N}-0 \mathrm{P}-0 \mathrm{~K}$ polymercoated urea fertilizer with $33.6 \%$ slow-release N (Polyon 42-0-0; Harrell's, Lakeland, FL)] was applied every 12 weeks based on the release rate of $\mathrm{N}$ curve determined from dissolution tests conducted by the manufacturer (Thornton Laboratories Testing and Inspection Services, Tampa, FL). Annual plant species received two $\mathrm{N}$ fertilizer applications at 0 and 12 weeks after planting (WAP) for a total $\mathrm{N}$ application of $1.38,2.31$, or $3.23 \mathrm{lb} / 1000 \mathrm{ft}^{2}$ over the course of the study (equivalent to an annual $\mathrm{N}$ rate of 3,5 , or $7 \mathrm{lb} /$ $1000 \mathrm{ft}^{2}$ ). Fifteen plants of each perennial species were transplanted in a rectangle configuration (a cluster of 3 plants $\times 5$ replicates) with $1-\mathrm{ft}$ spacing into each of nine raised-bed plots $(10 \times$ $20 \times 0.5 \mathrm{ft})$ and nine field plots $(10 \times$ $39 \mathrm{ft}$ ) on 4 Mar. 2011. Perennial plant species received five $\mathrm{N}$ fertilizer applications at $0,12,24,36$, and 48 WAP for a total $\mathrm{N}$ application of $1.15,3.46$, or $5.77 \mathrm{lb} / 1000 \mathrm{ft}^{2}$ over the course of the study (equivalent to an annual $\mathrm{N}$ rate of 1,3 , or $5 \mathrm{lb} /$ $1000 \mathrm{ft}^{2}$ ). All plant species were fertilized at three different $\mathrm{N}$ rates, not to exceed current recommendations for landscape fertilization (Florida Department of Environmental Protection, 2002). No mulch was added to raised beds or field plots to minimize outside $\mathrm{N}$ contributions.

Irrigation was applied to raised beds and field plots through nine drip lines (Jain Irrigation, Winter Haven, FL) that were spaced $1 \mathrm{ft}$ apart with emitters spaced 8 inches apart. The flow rate was $0.65 \mathrm{gal} / 100 \mathrm{ft}^{2}$ of drip tape. Annual species were irrigated two times per day for $45 \mathrm{~d}$ and then three times per week for the remainder of the study. Perennial species were watered twice daily for $9 \mathrm{~d}$, once daily for the next $12 \mathrm{~d}$, and three times per week for the remainder of the study. All irrigation events were $25 \mathrm{~min}$ in duration. Cumulative rainfall was 47.9 inches from Oct. 2010 to Mar. 2012 with average temperatures ranging from 51 to $81^{\circ} \mathrm{F}$, maximum temperatures of $96^{\circ} \mathrm{F}$, and minimum temperatures of $22{ }^{\circ} \mathrm{F}$ (University of Florida, 2014). Weeds were manually removed or treated with glyphosate (Round-Up ${ }^{\circledR}$; Monsanto, Creve Coeur, MO) as needed.

On 14 June 2011 (10 WAP), ageratum, salvia, and marigold planted in both locations were cut back to remove excessive growth and to restore shape. On 17 Nov. 2011 (37 WAP), blanket flower, goldenrod, and salvia (raised beds only) were cut back to remove excess growth and protect plants from cold damage; field grown salvia were cut back on 21 Dec. 2011 (42 WAP). Clippings were dried, weighed, and added to total shoot dry weight calculations.

SoIl Characterization. Before planting, soil samples were collected from the top 0 to 6 inches of each raised bed and field plot. Soil samples were air-dried, passed through a $2-\mathrm{mm}$ screen, and analyzed for soil $\mathrm{pH}$ (1:2 soil to deionized water ratio), organic matter (loss on ignition), and electrical conductivity (EC) by standard methods of the University of Florida Extension Soil Testing Laboratory (Mylavarapu, 2009). Mehlich 1 phosphorus $(\mathrm{P})$, potassium $(\mathrm{K})$, calcium $(\mathrm{Ca})$, and magnesium $(\mathrm{Mg})$ were analyzed using inductively coupled plasma-atomic emission spectroscopy [ICP-AES (Perkin Elmer, Waltham, MA)] following extraction using a 1:4 ratio of soil to $0.0125 \mathrm{M}$ sulfuric acid $\left(\mathrm{H}_{2} \mathrm{SO}_{4}\right)+0.05 \mathrm{M}$ hydrochloric acid $(\mathrm{HCl})$ (Mylavarapu, 2009). Colorimetric analysis of soil nitrate + nitrite $\left(\mathrm{NO}_{3}+\mathrm{NO}_{2}-\mathrm{N}\right)$ [U.S. Environmental Protection Agency (USEPA), 1993b] and 
ammonium $\left(\mathrm{NH}_{4}-\mathrm{N}\right)$ (USEPA, 1993a) was completed using a discrete analyzer (AQ2; Seal Analytical, Mequon, WI) following extraction for soil inorganic $\mathrm{N}$ (Mulvaney, 1996).

SHOOT DRY WEIGHT AND AESTHETIC QUALITY. Plant foliage was cut at the soil surface at 18 (annuals) or 54 (perennials) WAP and dried at $40.5{ }^{\circ} \mathrm{C}$ and weighed to determine shoot dry weight. Aesthetic quality of plants was rated on a scale of 0 to 5 (where $0=$ dead plant, $3=$ acceptable quality, $5=$ outstanding quality) by considering canopy density, dieback, flowers (when applicable), and general form (Shurberg et al., 2012a, 2012b). Quality ratings were conducted every 6 weeks for all annual and perennial landscape plants until plant harvest. Data were collected only on the center plants from each planting configuration (four annual plants and three perennial plants) to avoid edge effects.

Statistical analysis. The experiment was a split plot design with 18 raised-bed plots and 18 field plots. Three annual $\mathrm{N}$ rate treatments $(3,5$, or $7 \mathrm{lb} / 1000 \mathrm{ft}^{2}$ per year for annuals or 1,3 , or $5 \mathrm{lb} / 1000 \mathrm{ft}^{2}$ for perennials) were applied randomly to the raised bed plots and field plots with three replicates. Soil parameters were analyzed to determine differences in soil characteristics between field soils and subsoil fill using the PROC MIXED (SAS version 9.2; SAS Institute, Cary, NC) procedure in SAS with planting location (bed or field) as a fixed effect and plot as a random effect. Shoot dry weight was analyzed by species and WAP using the PROC MIXED (SAS version 9.2) procedure in SAS with $\mathrm{N}$ rate and planting location as fixed effects. Shoot dry weight analysis was conducted only for living plants; dead plants were considered missing data. Plant aesthetic quality data were analyzed using PROC GLIMMIX (SAS version 9.2) by species and WAP, with $\mathrm{N}$ rate and planting location as fixed effects. Nitrogen fertilizer rate and planting location main effects were described for most species, unless a significant $\mathrm{N}$ fertilizer $\times$ planting location interaction was noted.

Pairwise comparisons for shoot dry weight and aesthetic quality were completed using the Tukey's honestly significant difference test with a significance level of $\alpha=0.05$. Histogram and normality plots of the conditional residuals were examined to determine normality of the data.

\section{Results and discussion}

Soll Characterization. There were no differences between soil parameters among replicate beds or field plots. However, soil conditions varied significantly between raised beds containing subsoil fill and field plots containing native soil. The native field soils had significantly higher organic matter and Mehlich $1 \mathrm{~K}$ concentrations than the subsoil fill in the raised beds (Table 1). Subsoil fill in the raised beds had higher $\mathrm{pH}, \mathrm{EC}$, and Mehlich I P, Ca, and $\mathrm{Mg}$ concentrations than the native field soil (Table 1). Significant differences in initial soil $\mathrm{N}$ were noted for raised-bed subsoil fill and the native field soil. Mean initial soil $\mathrm{NO}_{3}+\mathrm{NO}_{2}-\mathrm{N}$ and $\mathrm{NH}_{4}-\mathrm{N}$ in the raised-bed plots were 1.22 and 2.69 $\mathrm{mg} \cdot \mathrm{kg}^{-1}$, respectively. In contrast, mean initial soil $\mathrm{NO}_{3}+\mathrm{NO}_{2}-\mathrm{N}$ and
$\mathrm{NH}_{4}-\mathrm{N}$ in the field plots were 3.95 and $1.46 \mathrm{mg} \cdot \mathrm{kg}^{-1}$, respectively.

SHOOT DRY WEIGHT. The warmseason annuals 'Cosmic Mix' and 'Cosmic Orange' cosmos and 'Saratoga Red' nicotiana died before 12 WAP regardless of planting location; 'Vista Red' salvia died before 18 WAP. Therefore, no dry weight data were available for these species. We do not attribute death of these species to $\mathrm{N}$ fertilization or planting location, but rather to plant stress resulting from mechanical damage to the tissue (possibly due to watering, weather conditions, or both).

There were few significant effects of $\mathrm{N}$ rate on annual or perennial shoot growth. Nitrogen fertilizer rate affected the shoot dry weight of dahlberg daisy, 'Survivor Hot Pink' geranium, and 'Mystic Spires' salvia. Dahlberg daisy and geranium produced greater shoot dry weight when they received $7 \mathrm{lb} / 1000 \mathrm{ft}^{2} \mathrm{~N}$ per year, compared with $3 \mathrm{lb} / 1000 \mathrm{ft}^{2} \mathrm{~N}$ per year, regardless of planting

Table 1. Mean soil properties for initial soil samples collected from raised beds containing subsoil fill material and native field soils used for evaluation of selected annual and perennial ornamental species response to nitrogen $(\mathbf{N})$ fertilization and planting conditions in west-central Florida (U.S. Department of Agriculture hardiness zone 9a).

\begin{tabular}{|c|c|c|}
\hline \multirow[b]{2}{*}{$\underline{\text { Soil test parameter }}{ }^{\mathrm{z}}$} & Raised beds & Field plots \\
\hline & \multicolumn{2}{|c|}{$($ mean $\pm S E)$} \\
\hline Organic matter $\left(\mathrm{g} \cdot \mathrm{kg}^{-1}\right)$ & $12.3 \pm 0.38 \mathrm{a}^{\mathrm{y}}$ & $23.4 \pm 0.60 \mathrm{~b}$ \\
\hline $\mathrm{pH}$ & $6.57 \pm 0.05 b$ & $5.67 \pm 0.03 \mathrm{a}$ \\
\hline $\mathrm{EC}\left(\mu \mathrm{S} \cdot \mathrm{cm}^{-1}\right)$ & $88.9 \pm 2.67 b$ & $61.2 \pm 2.72 \mathrm{a}$ \\
\hline Mehlich 1 phosphorus $\left(\mathrm{mg} \cdot \mathrm{kg}^{-1}\right)$ & $85.0 \pm 11.6 \mathrm{~b}$ & $52.9 \pm 9.31 \mathrm{a}$ \\
\hline Mehlich 1 potassium $\left(\mathrm{mg} \cdot \mathrm{kg}^{-1}\right)$ & $6.95 \pm 0.48 \mathrm{a}$ & $28.0 \pm 1.82 \mathrm{~b}$ \\
\hline Mehlich 1 calcium $\left(\mathrm{mg} \cdot \mathrm{kg}^{-1}\right)$ & $629 \pm 28.2 \mathrm{~b}$ & $455 \pm 26.9 \mathrm{a}$ \\
\hline Mehlich 1 magnesium $\left(\mathrm{mg} \cdot \mathrm{kg}^{-1}\right)$ & $67.3 \pm 4.01 \mathrm{~b}$ & $46.6 \pm 2.85 \mathrm{a}$ \\
\hline $\begin{array}{l}\text { Nitrate + nitrate }\left(\mathrm{NO}_{3}\right)-\mathrm{N}+\text { nitrite } \\
\quad\left(\mathrm{NO}_{2}\right)-\mathrm{N}\left(\mathrm{mg} \cdot \mathrm{kg}^{-1}\right)\end{array}$ & $6.97 \pm 1.27 b$ & $2.19 \pm 0.19 \mathrm{a}$ \\
\hline Ammonium $\left(\mathrm{NH}_{4}\right)-\mathrm{N}\left(\mathrm{mg} \cdot \mathrm{kg}^{-1}\right)$ & $2.73 \pm 0.4 / \mathrm{a}$ & $3.23 \pm 1$ \\
\hline
\end{tabular}

${ }^{2} 1 \mathrm{~g} \cdot \mathrm{kg}^{-1}=0.1 \%, \mathrm{l} \mu \mathrm{S} \cdot \mathrm{cm}^{-1}=1 \mu \mathrm{mho} / \mathrm{cm}, \mathrm{lmg} \cdot \mathrm{kg}^{-1}=1 \mathrm{ppm}$.

'Mean separation for each parameter (row) by Tukey's honestly significant difference test at $P<0.05$.

Table 2. Shoot dry weight response of landscape-grown dahlburg daisy, 'Survivor Hot Pink' geranium, and 'Mystic Spires' salvia to nitrogen (N) fertilizer applications at annual rates $\left(1,3,5\right.$, or $\left.7 \mathrm{lb} / 1000 \mathrm{ft}^{2}\right)$ when planted in west-central Florida (U.S. Department of Agriculture hardiness zone 9a).

\begin{tabular}{lccc}
\hline \multirow{2}{*}{$\begin{array}{c}\text { Annual fertilizer } \\
\mathbf{N} \text { rate }\left(\mathbf{l b} / \mathbf{1 0 0 0} \mathbf{f t}^{2}\right)^{\mathrm{z}}\end{array}$} & $\begin{array}{c}\text { Shoot wt }(\mathbf{g})^{\mathrm{z}} \\
\text { daisy }\end{array}$ & $\begin{array}{c}\text { 'Survivor Hot } \\
\text { Pink' geranium }\end{array}$ & $\begin{array}{c}\text { 'Mystic Spires' } \\
\text { salvia }\end{array}$ \\
\hline $\mathrm{l}$ & $\mathrm{N} / \mathrm{A}^{\mathrm{y}}$ & $\mathrm{N} / \mathrm{A}$ & $36.2 \mathrm{a}$ \\
3 & $31.4 \mathrm{a}^{\mathrm{x}}$ & $31.9 \mathrm{a}$ & $76.5 \mathrm{~b}$ \\
5 & $38.3 \mathrm{ab}$ & $35.6 \mathrm{ab}$ & $67.6 \mathrm{~b}$ \\
7 & $46.0 \mathrm{~b}$ & $43.3 \mathrm{~b}$ & $\mathrm{~N} / \mathrm{A}$ \\
\hline
\end{tabular}

${ }^{2} 1 \mathrm{lb} / 1000 \mathrm{ft}^{2}=48.8243 \mathrm{~kg} \cdot \mathrm{ha}^{-1}, 1 \mathrm{~g}=0.0353 \mathrm{oz}$.

N/A indicates that plant growth was not evaluated at that annual fertilizer $\mathrm{N}$ rate.

xMean separation for each species (column) by Tukey's honestly significant difference test at $P<0.05$. 
location (Table 2). 'Mystic Spires' salvia receiving 5 or $7 \mathrm{lb} / 1000 \mathrm{ft}^{2}$ of $\mathrm{N}$ per year produced greater shoot dry weight than plants supplied with 3 $\mathrm{lb} / 1000 \mathrm{ft}^{2}$ per year (Table 2). Shoot dry weight of other annual and perennial species evaluated was unaffected by $\mathrm{N}$ fertilizer rate (data not shown).

Shoot dry weight at 18 WAP was typically greater for surviving annual species planted in field soils when compared with plants grown in the raised beds containing lower-fertility subsoil fill material (Table 3). Only dusty miller plants produced greater shoot dry mass at 54 WAP when grown in raised beds than in field plots (Table 3). All perennial species, with the exception of mondo grass, produced greater shoot dry weight when grown in field plots as compared with raised beds containing subsoil fill (Table 3); mondo grass shoot dry weight was unaffected by planting location (data not shown).

A significant $\mathrm{N}$ fertilizer $\times$ planting location interaction was noted for 'Super Elfin Bright Orange' impatiens, 'Montego Yellow' or 'Montego Purple' snapdragon, and 'Sundial Yellow' moss rose. For these annual species, shoot dry weight at 18 WAP was greater for field grown plants that received 3 and/or $5 \mathrm{lb} / 1000 \mathrm{ft}^{2}$ of N annually, when compared with plants grown in raised beds containing subsoil fill material (Table 4 ). No significant $\mathrm{N}$ fertilizer $\times$ planting location effect was noted for any other cool- or warm-season annual or herbaceous perennial species.

Aesthetic Quality. With the exception of 'Survivor Hot Pink' geranium at $6 \mathrm{WAP}$ and 'Mystic Spires' salvia at $18,24,30$, and $54 \mathrm{WAP}, \mathrm{N}$ fertilizer rate had no effect on plant quality. 'Survivor Hot Pink' geranium in both planting locations fertilized with an annual $\mathrm{N}$ rate of $5 \mathrm{lb} /$ $1000 \mathrm{ft}^{2}$ had higher quality ratings (3.4 on average) than plants receiving $3 \mathrm{lb} / 1000 \mathrm{ft}^{2}$ of $\mathrm{N}$ annually (3.0). Similarly, 'Mystic Spires' salvia in both planting locations fertilized at the annual $\mathrm{N}$ rate of $1 \mathrm{lb} / 1000 \mathrm{ft}^{2}$ had a lower quality rating than plants receiving 3 and/or $5 \mathrm{lb} / 1000 \mathrm{ft}^{2}$ of $\mathrm{N}$ annually (Table 5).

In contrast, the quality of many annual (Table 6) and perennial (Table 7) species was influenced by planting location. When a significant planting location effect was noted,
Table 3. Shoot dry weight response of landscape-grown annual and perennial species to planting location (subsoil filled raised beds or native soil field plots) in west-central Florida (U.S. Department of Agriculture hardiness zone 9a).

\begin{tabular}{|c|c|c|}
\hline \multirow[b]{2}{*}{ Plant type and species } & \multicolumn{2}{|c|}{ Shoot wt $(g)^{z}$} \\
\hline & Raised beds & Field plots \\
\hline \multicolumn{3}{|l|}{ Cool-season annuals } \\
\hline Alyssum & $9.56 \mathrm{a}^{\mathrm{y}}$ & $16.7 \mathrm{~b}$ \\
\hline 'Bada Bing White' wax begonia & $24.4 \mathrm{a}$ & $42.7 \mathrm{~b}$ \\
\hline Dahlberg daisy & $26.5 \mathrm{a}$ & $50.7 \mathrm{~b}$ \\
\hline 'Survivor Hot Pink' geranium & $25.9 \mathrm{a}$ & $46.6 \mathrm{~b}$ \\
\hline Gomphrena & $8.43 \mathrm{a}$ & $20.7 \mathrm{~b}$ \\
\hline \multicolumn{3}{|l|}{ Warm-season annuals } \\
\hline $\begin{array}{l}\text { 'Blue Puffs Improved' ('Blue Danube') } \\
\text { ageratum }\end{array}$ & $20.5 \mathrm{a}$ & $32.6 \mathrm{~b}$ \\
\hline 'Silverdust' dusty miller & $39.3 \mathrm{~b}$ & $31.3 \mathrm{a}$ \\
\hline 'Bonanza Orange' marigold & $16.1 \mathrm{a}$ & $18.5 \mathrm{a}$ \\
\hline 'Profusion Cherry' zinnia & $21.1 \mathrm{a}$ & $22.9 \mathrm{a}$ \\
\hline \multicolumn{3}{|l|}{ Herbaceous perennials } \\
\hline Blanket flower & $41.2 \mathrm{a}$ & $319 \mathrm{~b}$ \\
\hline Goldenrod & $16.5 \mathrm{a}$ & $38.9 \mathrm{~b}$ \\
\hline Mondo grass & $11.9 \mathrm{a}$ & $17.0 \mathrm{a}$ \\
\hline 'Mystic Spires' salvia & $38.0 \mathrm{a}$ & $82.2 \mathrm{~b}$ \\
\hline
\end{tabular}

${ }^{\mathrm{z}} 1 \mathrm{~g}=0.0353 \mathrm{oz}$.

'Mean separation for each species (row) by Tukey's honestly significant difference test at $P<0.05$.

Table 4. Shoot dry weight recorded 18 weeks after planting (WAP) of landscapegrown 'Super Elfin Bright Orange' impatiens, 'Montego Yellow' or 'Montego Purple' snapdragon, and 'Sundial Yellow' moss rose to nitrogen $(\mathrm{N})$ fertilizer applications at three annual rates $\left(3,5\right.$, or $\left.7 \mathrm{lb} / 1000 \mathrm{ft}^{2}\right)$ and planting location (subsoil filled raised beds or native soil field plots) planted in west-central Florida (U.S. Department of Agriculture hardiness zone 9).

\begin{tabular}{|c|c|c|c|}
\hline \multirow[b]{2}{*}{$\begin{array}{l}\text { Planting location and } \\
\text { annual fertilizer } \mathrm{N} \text { rate } \\
\left(\mathrm{lb} / 1000 \mathrm{ft}^{2}\right)^{\mathrm{z}}\end{array}$} & \multicolumn{3}{|c|}{ Shoot wt $(g)^{z}$} \\
\hline & $\begin{array}{l}\text { 'Super Elfin } \\
\text { Bright Orange' } \\
\text { impatiens }\end{array}$ & $\begin{array}{c}\text { 'Montego Yellow'/ } \\
\text { 'Montego Purple' } \\
\text { snapdragon }\end{array}$ & $\begin{array}{c}\text { 'Sundial Yellow' } \\
\text { moss rose } \\
\end{array}$ \\
\hline \multicolumn{4}{|l|}{ Raised beds } \\
\hline 3 & $12.4 \mathrm{a}^{\mathrm{y}}$ & $20.1 \mathrm{a}$ & $5.14 \mathrm{a}$ \\
\hline 5 & $14.6 \mathrm{ab}$ & $27.2 \mathrm{ab}$ & $8.34 \mathrm{a}$ \\
\hline 7 & $24.0 \mathrm{ab}$ & $34.8 \mathrm{bc}$ & $6.77 \mathrm{a}$ \\
\hline \multicolumn{4}{|l|}{ Field plots } \\
\hline 3 & $24.0 \mathrm{~b}$ & $37.0 \mathrm{c}$ & $8.05 \mathrm{a}$ \\
\hline 5 & $24.5 \mathrm{~b}$ & $42.3 \mathrm{c}$ & $23.4 \mathrm{~b}$ \\
\hline 7 & $16.3 \mathrm{ab}$ & $41.8 \mathrm{c}$ & $8.2 \mathrm{a}$ \\
\hline
\end{tabular}

${ }^{2} 1 \mathrm{lb} / 1000 \mathrm{ft}^{2}=48.8243 \mathrm{~kg} \cdot \mathrm{ha}^{-1}, \mathrm{lg}=0.0353 \mathrm{oz}$.

'Mean separation for each column by Tukey's honestly significant difference test at $P<0.05$.

Table 5. Aesthetic quality response of landscape-grown 'Mystic Spires' salvia to nitrogen $(\mathrm{N})$ fertilizer applications at three annual rates $\left(1,3\right.$, or $\left.5 \mathrm{lb} / 1000 \mathrm{ft}^{2}\right)$ when planted in west-central Florida (U.S. Department of Agriculture hardiness zone 9a).

\begin{tabular}{|c|c|c|c|c|}
\hline \multirow{2}{*}{$\begin{array}{l}\text { Annual fertilizer } N \text { rate } \\
\left(1 \mathrm{~b} / 1000 \mathrm{ft}^{2}\right)^{\mathrm{z}}\end{array}$} & \multicolumn{4}{|c|}{ Aesthetic quality $(0-5 \text { scale })^{y}$} \\
\hline & $18 \mathrm{WAP}^{\mathrm{x}}$ & $24 \mathrm{WAP}$ & $30 \mathrm{WAP}$ & 54 WAP \\
\hline 1 & $3.3 \mathrm{a}^{\mathrm{w}}$ & $3.2 \mathrm{a}$ & $2.8 \mathrm{a}$ & $2.1 \mathrm{a}$ \\
\hline 3 & $4.1 \mathrm{~b}$ & $3.9 \mathrm{~b}$ & $3.4 \mathrm{~b}$ & $2.2 \mathrm{~b}$ \\
\hline 5 & $4.2 \mathrm{~b}$ & $3.9 \mathrm{~b}$ & $3.3 \mathrm{ab}$ & $2.9 \mathrm{~b}$ \\
\hline
\end{tabular}


Table 6. Aesthetic quality response of landscape-grown annual species to planting location (subsoil filled raised beds or native soil field plots) in west-central Florida (U.S. Department of Agriculture hardiness zone 9a).

\begin{tabular}{|c|c|c|c|c|c|c|}
\hline \multirow[b]{3}{*}{ Plant type and species } & \multicolumn{6}{|c|}{ Aesthetic quality $(0-5 \text { scale })^{\mathrm{z}}$} \\
\hline & \multicolumn{2}{|c|}{$6 \mathrm{WAP}^{\mathrm{y}}$} & \multicolumn{2}{|c|}{$12 \mathrm{WAP}$} & \multicolumn{2}{|c|}{$18 \mathrm{WAP}$} \\
\hline & $\begin{array}{c}\text { Raised } \\
\text { beds }\end{array}$ & $\begin{array}{l}\text { Field } \\
\text { plots }\end{array}$ & $\begin{array}{c}\text { Raised } \\
\text { beds }\end{array}$ & $\begin{array}{l}\text { Field } \\
\text { plots }\end{array}$ & $\begin{array}{c}\text { Raised } \\
\text { beds }\end{array}$ & $\begin{array}{l}\text { Field } \\
\text { plots }\end{array}$ \\
\hline \multicolumn{7}{|l|}{ Cool-season annuals } \\
\hline Alyssum & $3.4 \mathrm{a}^{\mathrm{x}}$ & $4.5 \mathrm{~b}$ & $3.7 \mathrm{a}$ & $3.0 \mathrm{a}$ & $3.3 \mathrm{~b}$ & $2.3 \mathrm{a}$ \\
\hline 'Bada Bing White' wax begonia & - $^{\mathrm{w}}$ & - & $3.3 \mathrm{a}$ & $4.2 \mathrm{~b}$ & $3.8 \mathrm{a}$ & $3.7 \mathrm{a}$ \\
\hline Gomphrena & $3.9 \mathrm{~b}$ & $2.1 \mathrm{a}$ & $0.0 \mathrm{a}$ & $1.3 \mathrm{~b}$ & $0.0 \mathrm{a}$ & $2.0 \mathrm{~b}$ \\
\hline 'Super Elfin Bright Orange' impatiens & - & - & $1.4 \mathrm{a}$ & $2.7 \mathrm{~b}$ & $2.2 \mathrm{a}$ & $2.9 \mathrm{a}$ \\
\hline 'Montego Yellow' /'Montego Purple' snapdragon & $3.4 \mathrm{a}$ & $3.6 \mathrm{a}$ & $3.2 \mathrm{a}$ & $3.1 \mathrm{a}$ & \multicolumn{2}{|c|}{ Interaction $^{\mathrm{v}}$} \\
\hline \multicolumn{7}{|l|}{ Warm-season annuals } \\
\hline 'Blue Puffs Improved' ('Blue Danube') ageratum & $2.8 \mathrm{a}$ & $3.8 \mathrm{~b}$ & $1.6 \mathrm{a}$ & $2.3 \mathrm{a}$ & $1.9 \mathrm{a}$ & $2.0 \mathrm{a}$ \\
\hline Saratoga Red' nicotiana & $1.9 \mathrm{a}$ & $2.8 \mathrm{~b}$ & - & - & - & - \\
\hline 'Vista Red' salvia & $2.0 \mathrm{a}$ & $2.0 \mathrm{a}$ & $0.6 \mathrm{a}$ & $0.7 \mathrm{a}$ & - & - \\
\hline 'Profusion Cherry' zinnia & $2.9 \mathrm{a}$ & $3.6 \mathrm{~b}$ & $1.3 \mathrm{a}$ & $1.6 \mathrm{a}$ & $2.3 \mathrm{a}$ & $2.3 \mathrm{a}$ \\
\hline
\end{tabular}

${ }^{\mathrm{z}} 0=$ dead plant; 5 = outstanding plant quality (dense leaf canopy, high quality flowers and no nutrient deficiencies or dieback).

${ }^{y} \mathrm{WAP}=$ week after planting.

${ }^{x}$ Mean separation for each species and WAP by Tukey's honestly significant difference test at $P<0.05$.

"Missing data (after 6 WAP indicates dead plants).

${ }^{\mathrm{V}}$ Interaction $=$ Main effects could not be evaluated because a significant $(P<0.05) \mathrm{N}$ fertilizer and planting location interaction was noted .

most species achieved higher quality ratings when grown in the native field soils. The only exceptions were gomphrena at $6 \mathrm{WAP}$, alyssum and dusty miller at 18 WAP (Table 6), and 'Mystic Spires' salvia at 42 WAP (Table 7), where quality of plants grown in the raised beds was higher than for the field grown counterparts.

A significant $\mathrm{N}$ fertilizer $\times$ planting location interaction was noted for 'Sundial Yellow' moss rose at 12 WAP, 'Montego Yellow' /'Montego Purple' snapdragon at $18 \mathrm{WAP}$, and blanket flower at 18, 24, 30, and 36 WAP. Field planted 'Sundial Yellow' moss rose receiving $5 \mathrm{lb} / 1000 \mathrm{ft}^{2}$ of $\mathrm{N}$ annually had higher mean quality ratings (2.8) than plants receiving $3 \mathrm{lb} / 1000 \mathrm{ft}^{2} \mathrm{~N}$ planted in the raised beds (1.3) at 12 WAP; no other significant $\mathrm{N}$ fertilizer $\times$ planting location effects were noted for this species (data not shown). In contrast, 'Montego Yellow'/'Montego Purple' snapdragon planted in raised beds and fertilized at the 3 and $5 \mathrm{lb} / 1000 \mathrm{ft}^{2}$ annual $\mathrm{N}$ rate (mean quality ratings of 3.8 and 3.9 , respectively) achieved higher quality at 18 WAP than plants grown in the field at the 5 and $7 \mathrm{lb} / 1000 \mathrm{ft}^{2}$ annual $\mathrm{N}$ rate (mean quality rating of 3.0). At 18 and $24 \mathrm{WAP}$, the quality of blanket flower planted in the raised beds receiving $5 \mathrm{lb} / 1000 \mathrm{ft}^{2}$ was similar to plants grown in the field plots at all three fertilization rates. However, by $30 \mathrm{WAP}$, the quality of field grown blanket flower had begun to decline in both the field plots and raised beds (Table 8).

We observed very few significant dry weight or quality responses to $\mathrm{N}$ rate for most annual or perennial species evaluated, in both the raised beds and field plots. Previous work reports that aesthetically pleasing annual and perennial species could be grown when fertilized at low $\mathrm{N}$ rates. For example, Wright et al. (2009) reported acceptable growth and quality of four annual species ['Cocktail Vodka' begonia, 'Red Hot Sally' salvia, 'Bonanza Yellow' marigold (Tagetes erecta), and 'Cooler Pink' vinca (Catharanthus roseus)] on a Groseclose silt loam soil in Virgina when fertilized with $\mathrm{N}$ at an annual rate of $4 \mathrm{lb} / 1000 \mathrm{ft}^{2}$ or less. Similarly, (Chen et al., 2011) found that annual $\mathrm{N}$ applications of 2 to $4 \mathrm{lb} / 1000 \mathrm{ft}^{2}$ were sufficient to produce acceptable growth and quality in Louisiana of seven perennial landscape species [cigar plant (Cuphea ignea), 'Stella de Oro' daylily
(Hemerocallis), 'Siskiyou Pink' gaura (Oenothera lindheimeri), 'New Gold' lantana (Lantana camara), mexican heather (Cuphea byssopifolia), purple coneflower (Echinacea purpurea), and 'Goldsturm' rudbeckia (Rudbeckia fulgida)] when grown in raised beds containing native topsoil $(56 \%$ sand, $31 \%$ silt, $13 \%$ clay, and $1 \%$ organic matter). Additionally, (Broschat et al., 2008) found that little to no fertilizer was needed for producing acceptable quality pentas (Pentas lanceolata), and 'Hendersoni' allamanda (Allamanda cathartica) on Margate fine sand soil in southern Florida.

On the basis of the results of our study, we suspect that soil conditions had a greater influence on shoot production and aesthetic quality of the tested ornamental species than $\mathrm{N}$ fertilization. Results of the initial soil analysis clearly indicated that the native field soils should provide a better growing environment (higher organic matter and soil test $\mathrm{K}$, lower salts and $\mathrm{pH}$ ) than the subsoil fill. Alsup and Trewatha (2006) compared growth (height and spread) and quality response of culitvars of 10 ornamental species [joseph's coat (Alternanthera dentata), ornamental pepper (Capsicum annumm), dianthus 
Table 7. Aesthetic quality response of landscape-grown herbaceous perennial species to planting location (subsoil filled raised beds or native soil field plots) in west-central Florida (U.S. Department of Agriculture hardiness zone 9a).

\begin{tabular}{|c|c|c|c|c|}
\hline \multirow{2}{*}{$\begin{array}{l}\text { Week after planting (WAP) and } \\
\text { planting location }\end{array}$} & \multicolumn{4}{|c|}{ Aesthetic quality $(0-5 \text { scale })^{\mathrm{z}}$} \\
\hline & Blanket flower & Goldenrod & Mondo grass & Salvia \\
\hline \multicolumn{5}{|l|}{$6 \mathrm{WAP}$} \\
\hline Raised beds & $3.7 \mathrm{a}^{\mathrm{y}}$ & $3.2 \mathrm{a}$ & $3.4 \mathrm{a}$ & $3.9 \mathrm{a}$ \\
\hline Field & $4.4 \mathrm{~b}$ & $3.2 \mathrm{a}$ & $3.2 \mathrm{a}$ & $3.7 \mathrm{a}$ \\
\hline \multicolumn{5}{|l|}{12 WAP } \\
\hline Raised beds & $3.0 \mathrm{a}$ & $3.1 \mathrm{a}$ & $3.1 \mathrm{a}$ & $2.8 \mathrm{a}$ \\
\hline Raised beds & Interaction $^{\mathrm{x}}$ & $3.1 \mathrm{a}$ & $2.1 \mathrm{~b}$ & $3.0 \mathrm{a}$ \\
\hline Field & & $2.7 \mathrm{a}$ & $1.7 \mathrm{a}$ & $4.7 \mathrm{~b}$ \\
\hline \multicolumn{5}{|l|}{$24 \mathrm{WAP}$} \\
\hline Raised beds & Interaction & $3.8 \mathrm{a}$ & $2.3 \mathrm{a}$ & Interaction \\
\hline Field & & $3.9 \mathrm{a}$ & $2.3 \mathrm{a}$ & \\
\hline \multicolumn{5}{|l|}{$30 \mathrm{WAP}$} \\
\hline Raised beds & Interaction & $1.0 \mathrm{a}$ & $2.4 \mathrm{a}$ & $2.2 \mathrm{a}$ \\
\hline Field & & $1.2 \mathrm{a}$ & $2.6 \mathrm{a}$ & $3.6 \mathrm{~b}$ \\
\hline \multicolumn{5}{|l|}{42 WAP } \\
\hline Raised beds & $1.1 \mathrm{a}$ & $1.2 \mathrm{a}$ & $2.2 \mathrm{a}$ & $2.6 \mathrm{~b}$ \\
\hline Field & $1.6 \mathrm{~b}$ & $1.6 \mathrm{a}$ & $2.3 \mathrm{a}$ & $2.2 \mathrm{a}$ \\
\hline \multicolumn{5}{|l|}{48 WAP } \\
\hline Raised beds & $2.3 \mathrm{a}$ & $1.9 \mathrm{a}$ & $2.1 \mathrm{a}$ & $2.6 \mathrm{a}$ \\
\hline Field & $3.1 \mathrm{a}$ & $2.2 \mathrm{a}$ & $2.2 \mathrm{a}$ & $2.2 \mathrm{a}$ \\
\hline \multicolumn{5}{|l|}{54 WAP } \\
\hline Raised beds & $2.4 \mathrm{a}$ & $2.6 \mathrm{a}$ & $1.9 \mathrm{a}$ & $2.0 \mathrm{a}$ \\
\hline Field & $3.2 \mathrm{a}$ & $2.9 \mathrm{a}$ & $1.7 \mathrm{a}$ & $2.7 \mathrm{a}$ \\
\hline
\end{tabular}

${ }^{\mathrm{z}} 0=$ dead plant; 5 = outstanding plant quality (dense leaf canopy, high quality flowers and no nutrient deficiencies or dieback).

yean separation for each species and WAP by Tukey's honestly significant difference test at $P<0.05$.

${ }^{\mathrm{x}}$ Interaction $=$ Main effects could not be evaluated because a significant $(P<0.05) \mathrm{N}$ fertilizer and planting location interaction was noted

Table 8. Aesthetic quality response of blanket flower to nitrogen (N) fertilizer applications at three annual rates $\left(1,3\right.$, or $\left.5 \mathrm{lb} / 1000 \mathrm{ft}^{2}\right)$ and planting location (subsoil filled raised beds or native soil field plots) at selected dates when planted in west-central Florida (U.S. Department of Agriculture hardiness zone 9).

\section{Planting location and annual fertilizer $\mathrm{N}$ rate $\left(\mathrm{lb} / 1000 \mathrm{ft}^{2}\right)^{\mathrm{z}}$}

\begin{tabular}{lcrr}
\multicolumn{4}{c}{ Aesthetic quality $(0-5)^{\mathrm{y}}$} \\
\hline $18 \mathrm{WAP}^{\mathrm{x}}$ & $24 \mathrm{WAP}$ & $30 \mathrm{WAP}$ & $36 \mathrm{WAP}$ \\
\hline
\end{tabular}

Raised beds

\begin{tabular}{lllll}
1 & $2.1 \mathrm{a}^{\mathrm{w}}$ & $2.3 \mathrm{a}$ & $2.4 \mathrm{ab}$ & $2.1 \mathrm{~b}$ \\
3 & $3.6 \mathrm{~b}$ & $3.0 \mathrm{~b}$ & $2.9 \mathrm{~b}$ & $3.2 \mathrm{c}$ \\
5 & $4.0 \mathrm{bc}$ & $4.0 \mathrm{c}$ & $2.8 \mathrm{~b}$ & $2.7 \mathrm{bc}$ \\
Field plots & & & & \\
1 & $4.7 \mathrm{c}$ & $4.0 \mathrm{c}$ & $2.6 \mathrm{ab}$ & $1.1 \mathrm{a}$ \\
3 & $4.8 \mathrm{c}$ & $4.4 \mathrm{c}$ & $1.9 \mathrm{a}$ & $1.1 \mathrm{a}$ \\
5 & $4.6 \mathrm{c}$ & $4.1 \mathrm{c}$ & $2.4 \mathrm{ab}$ & $1.4 \mathrm{a}$ \\
\hline
\end{tabular}

${ }^{\mathrm{z}} 1 \mathrm{lb} / 1000 \mathrm{ft}^{2}=48.8243 \mathrm{~kg} \cdot \mathrm{ha}^{-1}$.

${ }_{0} 0=$ dead plant; 5 = outstanding plant quality (dense leaf canopy, high quality flowers, and no nutrient deficiencies or dieback).

${ }^{x} \mathrm{WAP}=$ week after planting.

"Mean separation for each WAP by Tukey's honestly significant difference test at $P<0.05$.

(Dianthus chinensis), gazania (Gazania rigens), marigold, petunia (Petunia $\times$ bybrida), salvia, toothache plant (Spilanthes oleracea), verbena (Verbena $\times$ bybrida), and vinca] when grown in rocky, shallow field soils in Missouri and a purchased topsoil bag culture (similar to a raised bed). The authors reported that growth and quality response to soil conditions varied by cultivar, species, or both, with some plants growing taller or having higher quality when grown in the native soils compared with the bag culture. The authors suggested plants grown in the native soil had greater access to water and that the root substrate temperatures were lower when compared with the soil bags. Although we did not monitor soil moisture or root zone temperature, these conditions may have influenced the growth and quality of some of the species in our study. Small increases in water-holding capacity could have had marked effects on plant growth and quality due to the overall sandy, low water-holding capacity of central Florida soils.

Some of species evaluated in our study performed as well in the field (or better) as in the subsoil fill soils. Similarly, many of the cultivars evaluated by Alsup and Trewatha (2006) also performed as well or better in the soil bags when compared with the native field soil. These results illustrate that some landscape plant species are able to survive and thrive under various soil and fertility 
conditions. The subsoil fill used in this study was representative of material commonly used as "topsoil fill" in new residential landscape construction areas in west-central Florida. These "tougher" species may be good choices for installation in landscapes with marginal native soils or disturbed urban landscape soils. Future work might address the differences in plant growth based on water-holding capacities of these soils as well as the environmental benefits of changes in fertilizer application methods based on soil type and the economic benefit of using different $\mathrm{N}$ rates based on soil type.

\section{Literature cited}

Alsup, C.M. and P.B. Trewatha. 2006. Bagged soil tested as an alternative for growing bedding plants in the landscape. HortScience 41:1272-1275.

Broschat, T.K., D.R. Sandrock, M.L. Elliott, and E.F. Gilman. 2008. Effects of fertilizer type and quality and nutrient content of established landscape plants in Florida. HortTechnology 18:278-285.

Chen, Y., R.P. Bracy, A.D. Owings, and J.P. Quebedeaux. 2011. Controlledrelease fertilizer type and rate affect landscape establishment of seven herbaceous perennials. HortTechnology 21:336-342.
Florida Department of Environmental Protection. 2002. Best management practices for protection of water resources in Florida. Florida Dept. Environ. Protection, Tallahassee FL.

Israel, G.D. and G.W. Knox. 2001. Reaching diverse homeowner audiences with environmental landscape programs: Comparing lawn service users and nonusers. Univ. Florida Inst. Food Agr. Sci. AEC 363.

Line, D.E., N.M. White, D.L. Osmond, G.D. Jennings, and C.B. Mojonnier. 2002. Pollutant export from various land uses in the upper neuse river basin. Water Environ. Res. 74:100-108.

Mulvaney, R.L. 1996. NitrogenInorganic forms, p. 1123-1184. In: D.L. Sparks (ed.) Methods of soil analysis. Part 3: Chemical methods. Soil Sci. Soc. Amer., Madison, WI.

Mylavarapu, R.S. 2009. UF/IFAS Extension soil testing laboratory (ESTL) analytical procedures and training manual. Univ. Florida Inst. Food Agr. Sci. Circ. 1248.

Shober, A.L., K.A. Moore, G. Hasing, C. Wiese, G.C. Denny, and G.W. Knox. 2014. Effect of nitrogen fertilization rate on aesthetic quality of landscape-grown vines and groundcovers. HortTechnology 24:604-609.

Shober, A.L., K.A. Moore, N.G. West, C. Wiese, G. Hasing, G. Denny, and G.W. Knox. 2013. Growth and quality response of woody shrubs to nitrogen fertilization rates during landscape establishment in Florida. Hort Technology 23:898-904.
Shurberg, G., A.L. Shober, C. Wiese, G. Denny, G.W. Knox, K.A. Moore, and M.C. Giurcanu. 2012a. Growth and quality response of five landscape-grown herbaceous perennials to nitrogen fertilization at five rates. HortTechnology 22:787-797.

Shurberg, G., A.L. Shober, C. Wiese, G. Denny, G.W. Knox, K.A. Moore, and M.C. Giurcanu. 2012b. Response of landscape-grown warm- and cool-season annuals to nitrogen fertilization at five rates. HortTechnology 22:368-375.

U.S. Environmental Protection Agency. 1993a. Method 350.1. Determination of ammonia nitrogen by semi-automated colorimetry, EPA-600/4-79-020. Environ. Monitoring Systems Lab., Office Res. Dev., U.S. Environ. Protection Agency, Cincinnati., $\mathrm{OH}$.

U.S. Environmental Protection Agency. 1993b. Method 353.2. Determination of nitrate-nitrite nitrogen by automated colorimetry. Environ. Monitoring Systems Lab., Office Res. Dev., U.S. Environ. Protection Agency, Cincinnati, OH.

University of Florida. 2014. Florida automated weather network, report generator. 5 Aug. 2014. <http://fawn.ifas.ufl.edu/ data/reports/>.

Wright, R.D., B.E. Jackson, M.C. Barnes, and J.F. Browder. 2009. The landscape performance of annual bedding plants grown in pine tree substrate. HortTechnology 19:78-82. 